\title{
Successful immune tolerance induction to enzyme replacement therapy in CRIM-negative infantile Pompe disease
}

\author{
Yoav H. Messinger, MD1, Nancy J. Mendelsohn, MD², William Rhead, MD, PhD³, David Dimmock, MD, \\ Eli Hershkovitz, MD', Michael Champion, MBBS ${ }^{5}$, Simon A. Jones, MBChB, BSc ${ }^{6}$, Rebecca Olson, RN, CNP², \\ Amy White, $\mathrm{MS}^{3}$, Cara Wells, $\mathrm{MS}^{3}$, Deeksha Bali, PhD7, Laura E. Case, DPT, PCS, C/NDT \\ Sarah P. Young, $\mathrm{PhD}^{7}$, Amy S. Rosenberg, $\mathrm{MD}^{9}$ and Priya S. Kishnani, MD ${ }^{7}$
}

\begin{abstract}
Purpose: Infantile Pompe disease resulting from a deficiency of lysosomal acid $\alpha$-glucosidase (GAA) requires enzyme replacement therapy (ERT) with recombinant human GAA (rhGAA). Crossreactive immunologic material negative (CRIM-negative) Pompe patients develop high-titer antibody to the rhGAA and do poorly. We describe successful tolerance induction in CRIM-negative patients.
\end{abstract}

Methods: Two CRIM-negative patients with preexisting anti-GAA antibodies were treated therapeutically with rituximab, methotrexate, and gammaglobulins. Two additional CRIM-negative patients were treated prophylactically with a short course of rituximab and methotrexate, in parallel with initiating rhGAA.

Results: In both patients treated therapeutically, anti-rhGAA was eliminated after 3 and 19 months. All four patients are immune tolerant to rhGAA, off immune therapy, showing B-cell recovery while continuing to receive ERT at ages 36 and 56 months (therapeutic) and 18 and 35 months (prophylactic). All patients show clinical response to ERT, in stark contrast to the rapid deterioration of their nontolerized CRIM-negative counterparts.

Conclusion: The combination of rituximab with methotrexate \pm intravenous gammaglobulins (IVIG) is an option for tolerance induction of CRIM-negative Pompe to ERT when instituted in the naïve setting or following antibody development. It should be considered in other conditions in which antibody response to the therapeutic protein elicits robust antibody response that interferes with product efficacy.

Genet Med 2012:14(1):135-142

Key Words: immune tolerance; methotrexate; Pompe disease; rituximab

\section{INTRODUCTION}

Infantile Pompe disease (OMIM\# 232300) is a fatal disease resulting from a deficiency of lysosomal acid $\alpha$-glucosidase (GAA). ${ }^{1}$ Enzyme replacement therapy (ERT) with recombinant human acid $\alpha$-glucosidase (rhGAA) is the sole disease-specific treatment currently available. Patients with two deleterious mutations and complete absence of GAA, as assessed by western blot, are classified as cross-reactive immunologic material negative (CRIM-negative). Patients with GAA protein detectable by western blot are classified as CRIM-positive. ${ }^{2-4}$ Whereas the majority of CRIM-positive patients have sustained therapeutic responses to ERT, CRIM-negative patients almost uniformly do poorly, experiencing rapid clinical decline because of the development of sustained, high-titer antibodies to rhGAA. ${ }^{4}$ CRIM-negative patients therefore serve as an excellent model to evaluate the impact of therapies aimed at immune tolerance.
We reported the first successful reversal of rhGAA antibodies in a CRIM-negative Pompe patient treated with rituximab, intravenous gammaglobulins (IVIG), and methotrexate. ${ }^{5}$ We now report that this patient and an additional CRIM-negative patient treated similarly are indeed immune tolerant. Critically, such tolerance can be induced prophylactically, commencing with ERT, using a short rituximab with methotrexate regimen, thereby avoiding prolonged immune suppression. The two prophylactically treated patients and two therapeutically treated patients remain tolerant to continued administration of rhGAA, off of all immune therapy and with recovered B cells. All patients have achieved motor gains, in contrast to the relentless downhill course of nontolerant ERT-treated CRIMnegative patients. However, like some CRIM-positive patients, patients are left with residual deficiencies not reversible by ERT due to preexisting damage prior to the start of ERT.

\footnotetext{
${ }^{1}$ Pediatric Hematology/Oncology, Children's Hospitals and Clinics of Minnesota, Minneapolis, Minnesota, USA; ${ }^{2}$ Medical Genetics, Children's Hospitals and Clinics of Minnesota, Minneapolis, Minnesota, USA; ${ }^{3}$ Division of Genetics, Departments of Pediatrics, Medical College of Wisconsin and Children's Hospital of Wisconsin, Milwaukee, Wisconsin, USA; ${ }^{4}$ Pediatric Endocrinology and Metabolism Unit, Soroka Medical Center, Beer Sheva, Israel; ${ }^{5}$ Department of Inherited Metabolic Disease, Evelina Children's Hospital, London, UK; ${ }^{6}$ Genetic Medicine, Manchester Academic Health Science Centre, Central Manchester University, Manchester, UK; ${ }^{7}$ Division of Medical Genetics, Department of Pediatrics, Duke University Medical Center, Durham, North Carolina, USA; ${ }^{8}$ Division of Physical Therapy, Department of Community and Family Medicine, Duke University Medical Center, Durham, North Carolina, USA; ${ }^{\circ}$ Division of Therapeutic Proteins, Center for Drug Evaluation and Research, US Food and Drug Administration, Bethesda, Maryland, USA.

Correspondence: Yoav H. Messinger (yoav.messinger@childrensmn.org)
} 


\section{PATIENTS AND METHODS}

This multinational collaborative effort received individual institutional review board or ethics committee approval. In all cases, motor assessment, cardiac assessment, and other clinical parameters were obtained from medical records. GAA mutation analysis was determined as previously described. ${ }^{6}$ CRIM status was determined as previously described. ${ }^{2}$ Briefly, cell lysates derived from the patient's fibroblasts were subjected to western blot analysis in a single laboratory with a polyclonal antibody that was made against human placental GAA, which recognizes all GAA protein forms. A patient is considered CRIM-negative if no GAA is detected in the western blot assay and the patients have deleterious mutations in the GAA gene. IgG antibodies to rhGAA were measured using enzyme-linked immunosorbent assays and confirmed using radioimmunoprecipitation, as previously described. ${ }^{6}$ Urinary glucose tetrasaccharide $\left(\mathrm{Glc}_{4}\right)$ level was determined as the total hexose tetrasaccharide fraction in urine measured by high-pressure liquid chromatography with ultraviolet detection and electrospray ionization-tandem mass spectrometry as previously described ${ }^{7}$. Flow cytometry was used to assess CD19-positive (B cell) percentage, using standard techniques at each local institution.

rhGAA (Myozyme, Genzyme, Cambridge, MA) administered every 2 weeks was initiated after diagnosis of Pompe in all cases. ${ }^{2,3}$ Dosing ranged from 20 to $40 \mathrm{mg} / \mathrm{kg}$ every 2 weeks.

Patients 1 and 2 were treated therapeutically by rituximab, methotrexate, and IVIG after the development of rhGAA antibodies, until antibodies were eliminated. Rituximab $375 \mathrm{mg} / \mathrm{m}^{2} /$ dose was given weekly for 4 weeks followed by maintenance dosing. Methotrexate $0.5 \mathrm{mg} / \mathrm{kg}$ weekly was given enterally, based on hematologic tolerance. IVIG $0.5 \mathrm{~g} / \mathrm{kg}$ was given every 4 weeks (Figure 1a,b). Patients 3 and 4 were treated prophylactically with a short course of rituximab and methotrexate. Rituximab $375 \mathrm{mg} / \mathrm{m}^{2} /$ dose was administered weekly for 4 weeks (the first dose given 1 day before the first rhGAA administration), and methotrexate $0.4 \mathrm{mg} / \mathrm{kg}$ was given subcutaneously 3 times a week for 9-17 doses (Figure 2a,b).

\section{RESULTS}

Therapeutic Tolerance Induction for an Established Antibody Patients 1 and 2 presented with hypotonia, cardiomyopathy, elevated creatinine phosphokinase (CPK) level, and elevated urine $\mathrm{Glc}_{4}$ level at ages 5 weeks and 12 days, respectively. Both were confirmed as CRIM-negative Pompe patients (Table 1). rhGAA was initiated soon after diagnosis, at 7 weeks and 16 days of age, respectively (Table 1). IgG antibodies to rhGAA were detected after 4-6 weeks, escalating to maximum titers of 1:1,600 and 1:12,800, respectively. An immune tolerance regimen of rituximab, methotrexate, and IVIG was initiated as shown in Figure 1. Antibodies to rhGAA were fully eliminated 3 months after commencement of immune therapy in patient 1 (Figure 1a) and after 19 months in patient 2 (Figure 1b), although there was a rapid drop in titer following the first course. Patient 1 is tolerant to rhGAA after more than 4.5 years of ERT and patient 2 after 3 years of ERT. Both are off all immune therapy, with
B-cell recovery (Figure 1). The immune tolerance regimen was very well tolerated with mild intermittent neutropenia requiring withholding methotrexate and only mild viral infections. Although patient 1 became ventilator dependent after 11 months of ERT, he continued to improve, now requiring ventilation only at night, whereas patient 2 was not ventilated (Table 2). All medications including rhGAA, rituximab, and IVIG were given at home to patient 1 , under strict monitoring by skilled nursing staff. In both patients, cardiomyopathy rapidly resolved after initiating rhGAA (Table 2). Patients 1 and 2, diagnosed and treated before 2 months of age, had high baseline urinary $\mathrm{Glc}_{4}$ values, which then decreased to less than those of CRIMnegative patients who did not receive immune tolerance therapy (Figure 3). Clinically, both patients continue to gain developmental milestones. Even though patient 1 has significant motor and speech deficits, he continues to improve, whereas patient 2 has only mild deficits (Table 2). Hearing tests were initially normal in both patients, but patient 1 developed mild conductive hearing loss in the middle-to-high frequencies at the age of 56 months, and patient 2 required pressure-equalizing tubes at the age of 26 months. At the age of 54 months, magnetic resonance imaging (MRI) of the brain of patient 1 revealed new, extensive deep white matter changes, sparing the subcortical U-fibers (Figure 4), whereas MRI of the brain of patient 2 (age 31 months) was normal (Table 2). Patient 1 is receiving rhGAA every 2 weeks to date, patient 2 received rhGAA every 2 weeks, but after 29 months of ERT, rhGAA frequency was increased to every week due to increased creatinine phosphokinase (CPK) levels, progression of ptosis, increased fatigue, and decreased stamina, all of which have since improved.

\section{Prophylactic Tolerance Induction}

Patients 3 and 4 presented with hypotonia, cardiomyopathy, and elevated creatinine phosphokinase (CPK) level at the ages of 8 and 10 weeks, respectively. They were confirmed as CRIMnegative Pompe patients (Table 1) and received the toleranceinduction regimen simultaneously with rhGAA at 15-16 weeks of age (Table 1, Figure 2). Patient 3 also received one dose of IVIG at week 4 due to a febrile illness. Both patients continued to receive rhGAA without further immune therapy. Patient 3 developed a transient anti-rhGAA antibody response, which reached a maximal titer of 1:1,600 and spontaneously declined to 1:200 after 24 months on ERT (Figure 2a) without additional immune tolerance therapy. In patient 3 , who at diagnosis had significant cardiac involvement with poor function, cardiomyopathy improved after 14 months of ERT, both in function and wall thickness, but did not completely resolve (Table 2). Ventilation was not required, and she continues to gain developmental milestones (Table 2). Commensurate with her excellent clinical course, $\mathrm{Glc}_{4}$ levels of patient 3 remain lower than those of nontolerized CRIM-negative patients (Figure 3). Patient 4 was persistently antibody negative (Figure $2 \mathrm{~b}$ ) and required only transient ventilation for 3 days at diagnosis. At diagnosis, he had significant cardiac involvement-dilated cardiomyopathy with very poor function. Although the LV mass improved, 

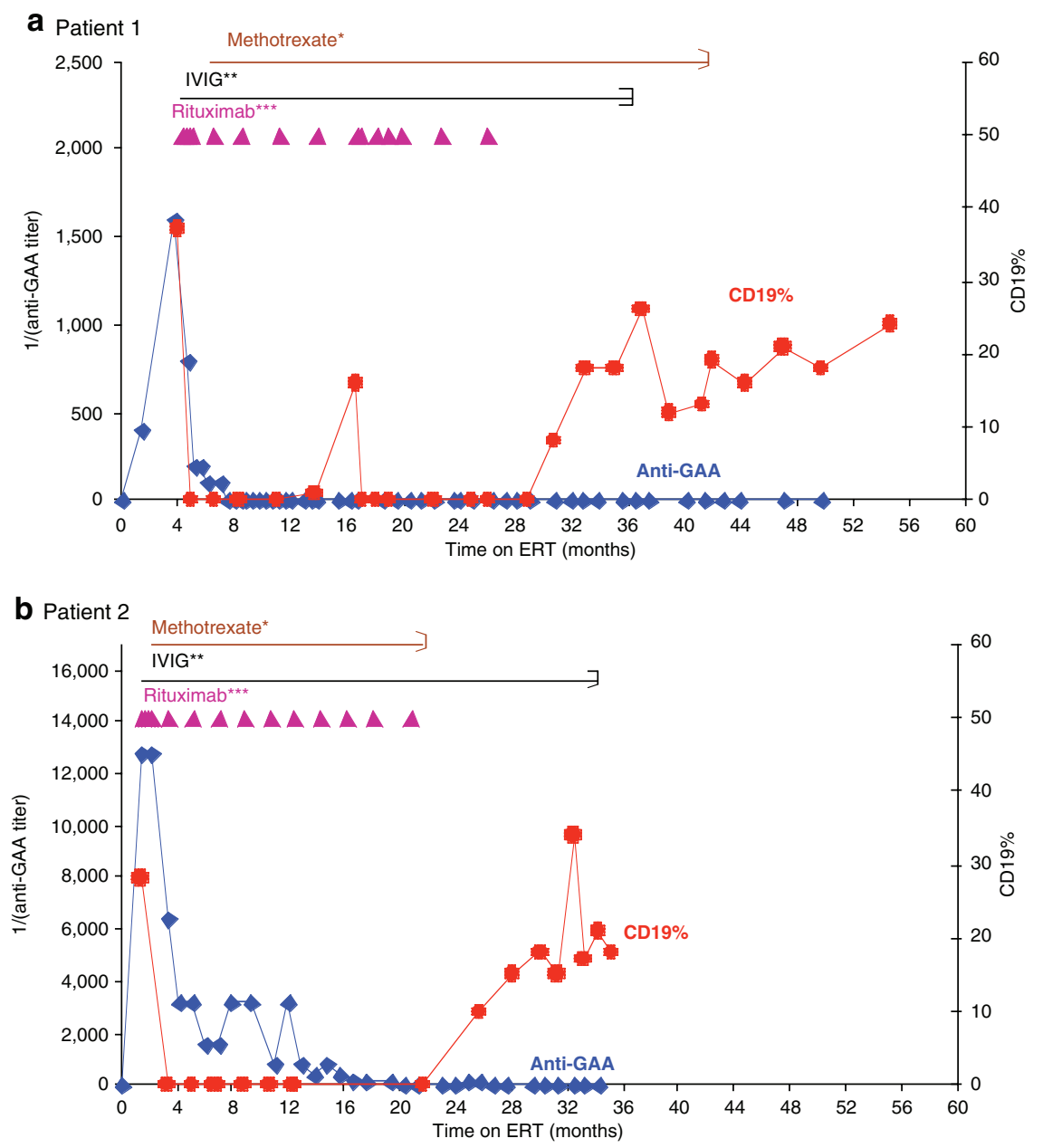

*Methotrexate $0.5 \mathrm{mg} / \mathrm{kg}$ weekly enterally
${ }^{* \star}$ IVIG: Immune globulins $0.5 \mathrm{~g} / \mathrm{kg}$ every 4 weeks
${ }^{* \star *}$ Rituximab $375 \mathrm{mg} / \mathrm{m}^{2} /$ dose IV

Figure 1 Therapeutic-treated patients with antibody to ERT. Tolerance-induction regimen, with rituximab, methotrexate, and intravenous gammaglobulins (IVIG) showing antibody levels and B-cell percentage for patients treated therapeutically for an established antibody response. Both patients achieved tolerance to ongoing enzyme replacement therapy, and are off immune therapy, with negative antibody titer showing B-cell recovery. (a) Patient 1. (b) Patient 2. ERT, enzyme replacement therapy.

the function to date has not changed (Table 2). He attained developmental milestones, but a parainfluenza infection at the age of 14 months resulted in neuromotor regression. His overall worse disease status is reflected in high $\mathrm{Glc}_{4}$ levels, which are currently comparable to those of nontolerized CRIM-negative patients (Figure 3). Patient 3 had normal MRI of the brain at the age of 17 months and an inconclusive hearing test at 12 months (which was not repeated), whereas patient 4 did not have an MRI or a formal hearing test (Table 2).

\section{DISCUSSION}

The clinical use of therapeutic enzymes has expanded rapidly in the past decade for a variety of disorders, with ERTs now available for several previously untreatable diseases. Therapeutic enzymes are potentially immunogenic, evoking antibody responses that may be without overt clinical significance or may lead to hypersensitivity reactions, decreased bioavailability, or decreased efficacy. ${ }^{8,9}$ Antibody-mediated abrogation of efficacy of the therapeutic proteins has been described in patients with other enzymeand factor-deficiency states such as mucopolysaccharidosis types I, II, and VI, Fabry disease, and severe hemophilia A and B. ${ }^{10-14}$

Most Pompe disease patients develop antibodies to ERT, but with dramatically different outcomes, depending on CRIM status. Antibody titers in the majority of CRIM-positive patients diminish over time and do not appear to neutralize efficacy, leaving patients with a favorable clinical response. In contrast, CRIM-negative patients and a subset of CRIM-positive patients mount high-titer and sustained antibody responses 
ORIGINAL RESEARCH ARTICLE

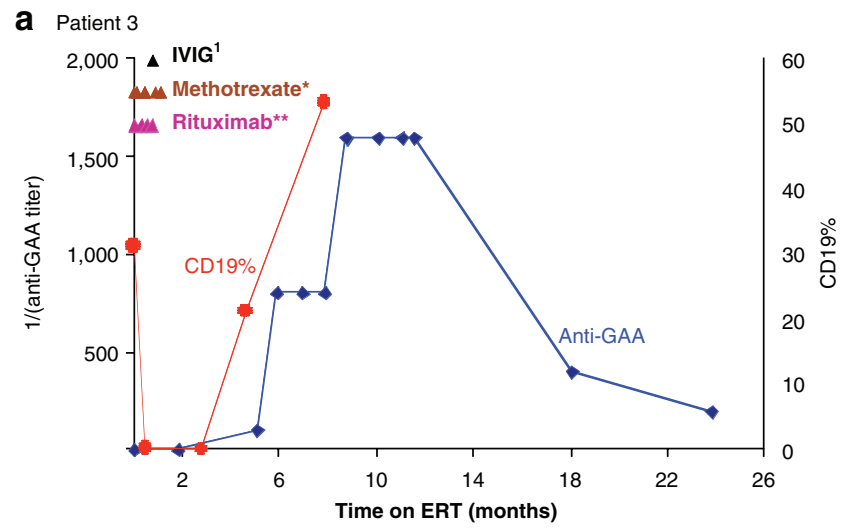

b Patient 4

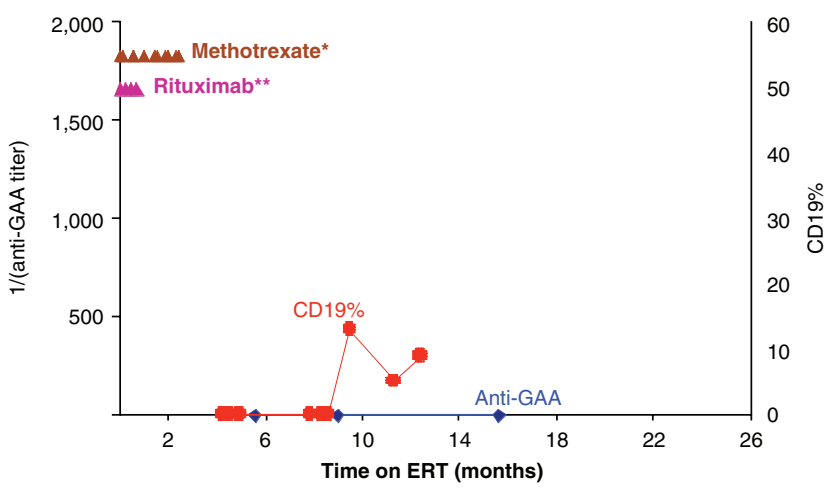

*Methotrexate $0.4 \mathrm{mg} / \mathrm{kg} \mathrm{SQ}$ x 3 days, every other week (patient 1-9 doses, patient 2-17 doses)

${ }^{* *}$ Rituximab $375 \mathrm{mg} / \mathrm{m}^{2} /$ dose IV weekly x 4 . First dose 1 day before rhGAA. ${ }^{1}$ Immune globulins $0.5 \mathrm{~g} / \mathrm{kg}$ once only patient 1

Figure 2 Prophylactic-treated patients. Tolerance-induction regimen, antibody levels, and B-cell percentage for patients treated prophylactically in the naïve setting with rituximab and methotrexate. Both patients are tolerant to ongoing enzyme replacement therapy, off immune therapy with B-cell recovery. (a) Patient 3 with remains with low-titer (1:200) antibody level, similar to CRIM-positive patients. (b) Patient 4 with negative antibody titer.

that neutralize enzyme uptake into cells with resulting poor outcome: death or invasive ventilation in patients by the age of 27 months. ${ }^{4,15}$ Therefore, there is clearly a need for immune tolerance induction to ERT in CRIM-negative Pompe patients and those CRIM-positive patients who are at risk for development of high sustained antibody titers.

We have previously described patient $1,{ }^{5}$ who was treated for an established antibody response, and now confirm his immune tolerance to the foreign protein. Remarkably, this patient and an additional patient treated for established antibody are off all immune suppressive therapy, have B-cell recovery, and are tolerant to rhGAA. Both patients had no significant toxicity or increased infections from this regimen, with the exception of transient leukopenia from methotrexate. Importantly, both patients began immune tolerance therapy relatively early after detection of an antibody response to rhGAA prior to development of a very high titer and sustained response. It is certainly possible that patients with very high persistent antibody titers (more than 1:51,200) may not respond to this regimen. ${ }^{15}$
MESSINGER et al | Immune tolerance to enzyme replacement in CRIM-negative Pompe

To assess whether a short course of immune tolerance induction therapy at commencement of ERT (thus avoiding prolonged immune suppression) would confer lasting tolerance, two additional CRIM-negative patients were treated prophylactically with a short regimen of rituximab and methotrexate essentially without IVIG. Remarkably, both patients display ongoing tolerance to ERT: patient 3 developed a transient antibody response that declined over time, similar to that seen in CRIM-positive patients, tolerating ERT at the age of 35 months with clinical gains. Patient 4 did not develop rhGAA antibodies but has a dilated cardiomyopathy and neuromotor deficits.

This regimen was chosen for tolerance induction in both therapeutic and prophylactic settings on the basis of data from clinical trials and experimental data. Rituximab has been used successfully in immune-mediated diseases, such as systemic lupus erythematosus ${ }^{16}$ as well as hemophilia patients with hightiter inhibitors. ${ }^{17}$ In patients with severe factor VIII deficiency with high-titer inhibitors, rituximab induced tolerance to therapeutic factor VIII with a variable degree of success. ${ }^{17-20}$ The mechanism by which rituximab induces tolerance is unknown but, in some circumstances, appears to be related to restoration of $\mathrm{T}$ regulatory cells. ${ }^{21}$ Regardless of mechanism, the success of the approach suggests that B cells are critical in the induction and maintenance of the immune response.

Interestingly, a recently reported CRIM-negative patient received another monoclonal agent, omalizumab, an anti-IgE, to prevent anaphylaxis to rhGAA. Strikingly, this patient has very low-titer IgG against rhGAA (1:400) after 44 months of ERT.2 ${ }^{22}$ This suggests some heterogeneity among CRIM-negative patients in the nature of the immune response to rhGAA as well as a novel use of anti-IgE.

One of the concerns with rituximab therapy is the profound B-cell suppression leading to decreased serum Ig levels and the heightened potential for adverse outcomes. Patient 1 failed to respond to routine vaccination administered in the treatment period and even after B-cell recovery (data not shown), suggesting the potency of this regimen. It suggests that following successful treatment, vaccination response should be monitored. To decrease infectious complications, both patients who had received prolonged rituximab therapy were placed on chronic IVIG. Additionally, it is well known that IVIG is also an immunomodulatory agent used extensively in the setting of autoimmune disease ${ }^{23,24}$; and thus, it is probable that the IVIG had dual effects in our patients: contributing to immune modulation and protecting against infectious agents.

Prolonged rituximab therapy with B-cell suppression has been associated with the development of progressive multifocal leukoencephalopathy (PML). ${ }^{25}$ Patient 1 developed changes of the brain affecting the deep white matter (detected on MRI; Figure4), which are clearly different from what is seen in progressive multifocal leukoencephalopathy that affect the subcortical area. ${ }^{25}$ Additionally, this patient shows slow progressive neurologic improvement rather than the clinical deterioration seen in progressive multifocal leukoencephalopathy. ${ }^{25}$ Thus this patient does not have PML. Since these MRI changes are similar to a 
Table 1 Baseline and immunologic data for patients treated therapeutically (patients 1 and 2) for an established antibody response as well as patients treated prophylactically (patients 3 and 4) in parallel to starting enzyme replacement therapy

Therapeutic tolerance induction

Prophylactic tolerance induction

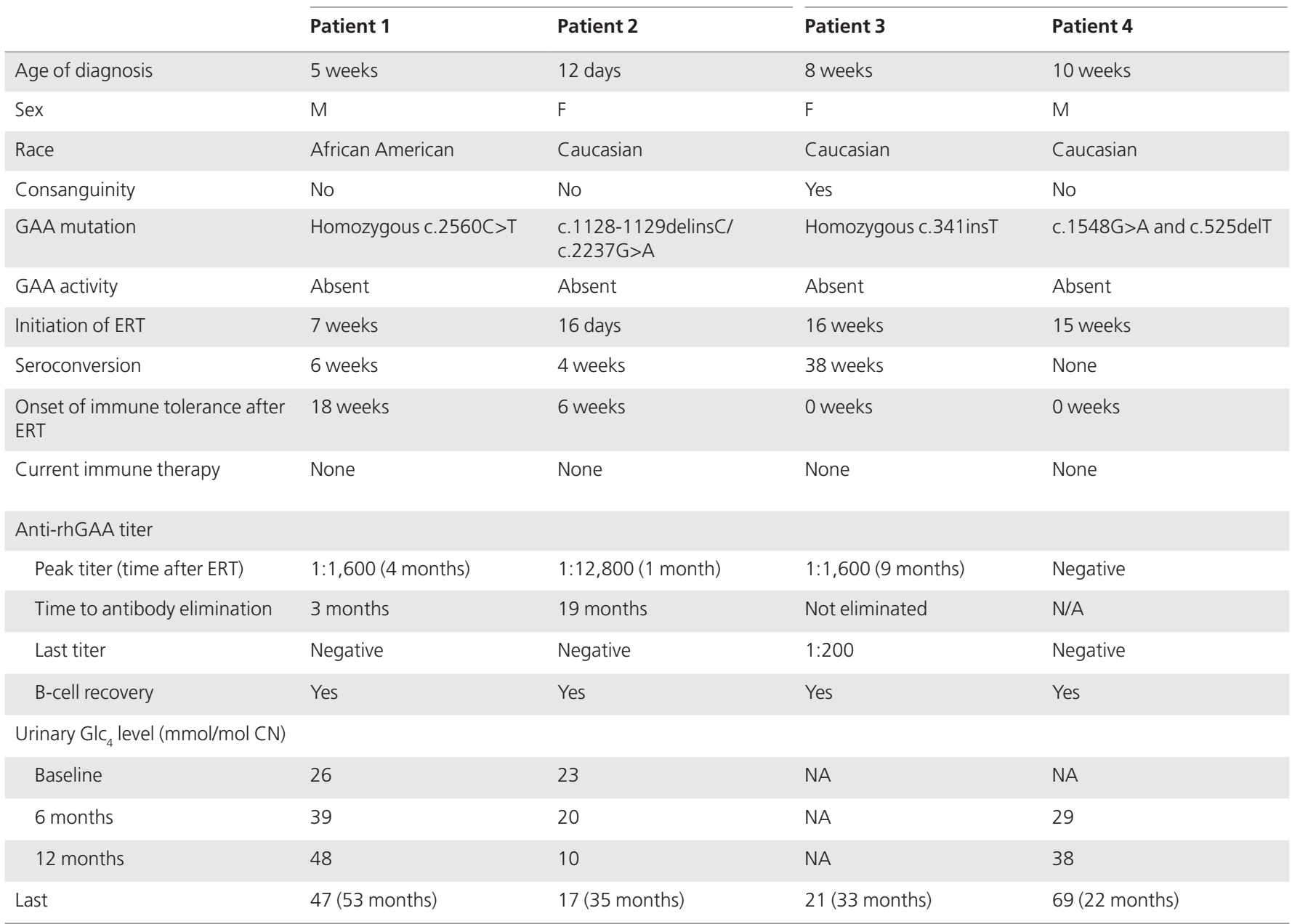

ERT, enzyme replacement therapy; GAA, acid $\alpha$-glucosidase; Glc4, glucose tetrasaccharide; rhGAA, recombinant human GAA.

previously described CRIM-negative patient, they may reflect the natural history of CRIM-negative Pompe disease. ${ }^{22}$

Finally, methotrexate was added to the regimen because it eliminates dividing lymphocytes, theoretically eliminating rhGAA-specific T cells and residual B cells. Methotrexate also minimized antibody to rhGAA when used as a sole agent in a mouse model of Pompe disease. ${ }^{26}$

Remarkably, all tolerized patients showed motor improvement, in stark contrast to ongoing deterioration of CRIMnegative patients, confirming the role of the immune response in abrogating effectiveness of ERT. ${ }^{4}$ Nonetheless, patients were left with residual deficiencies either due to irreversible changes or due to the inability of rhGAA to fully address disease manifestations. Patient 3 is left with mild cardiomyopathy, and patient 4 continues to have significant residual dilated cardiomyopathy despite immune tolerance to ERT. Both patients were diagnosed and started therapy at a later age, and it is possible that irreversible myocardial damage occurred prior to the start of ERT. Patient 1, at 56 months, has shown a clinical response to ERT and continues to make motor and developmental progress; however, hehas motor and speech delays. His pulmonary status has improved from full-time ventilation to requiring ventilator support only at night. The poorest outcome was in patient 4, who at the start of ERT already had significant motor involvement. Following appreciable motor improvement, this patient had motor deterioration with elevated urinary $\mathrm{Glc}_{4}$ level after a parainfluenza infection, thus illustrating the fragility of such patients. This outcome suggests that early identification of infantile Pompe disease, ideally via newborn screening, may abrogate these irreversible changes. It is critical to note that even patients 1 and 4, who had significant deficits, continue to have neuromotor improvement in contrast to the ongoing deterioration of CRIM-negative patients with high-titer antibody., ${ }^{4,15}$

The deficits seen in patients 1 and 4 and the elevated Glc level of patient 4 may be secondary to basic limitations of ERT in Pompe disease. These include the high levels of rhGAA required to achieve efficacy-approximately 30 -fold to 100 -fold greater than doses for ERT in other lysosomal disorders ${ }^{27}$-and the well-recognized variability of response by skeletal muscle. Potential factors involved in this variability include a lower 
Table 2 Outcome clinical data

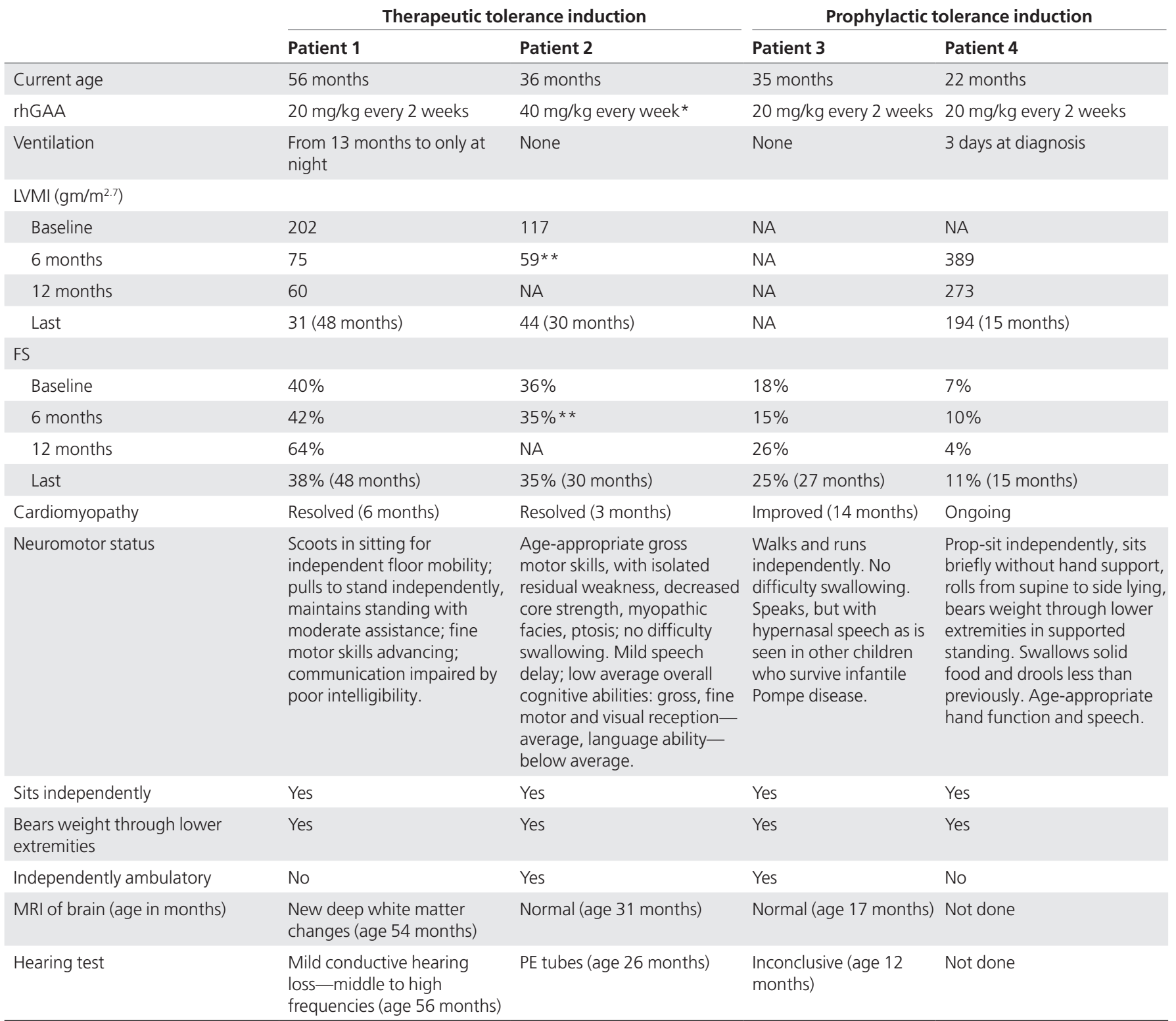

ERT, enzyme replacement therapy FS, fractional shortening by m-mode echocardiography; LVMI, left ventricular mass index per height ${ }^{2.7}$ ( $g m / m^{2.7}$ ); MRI, magnetic resonance imaging; NA, not available; PE tubes, pressure-equalizing tubes; rhGAA, recombinant human acid $\alpha$-glucosidase; CPK, creatinine phosphokinase.

*Patient 2 received rhGAA 20 mg/kg, which was changed to 30 mg/kg after 17 months of ERT and then to 40 mg/kg after 23 months. Frequency was increased to weekly 40 mg/ $\mathrm{kg}$ after 29 months of ERT due to increased CPK levels, progression of ptosis, increased fatigue, and decreased stamina, all of which have since improved.

**Patient 2 echocardiography data are for 9 months of ERT. No data for 6 or 12 months.

number of mannose-6-phosphate receptors (the principal receptors for enzyme uptake and lysosomal targeting) in skeletal muscle in comparison to the heart, inefficient targeting to skeletal muscle, accumulated muscle and lysosomal damage, and resistance to correction by type II myofibers.

In conclusion, strategic use of rituximab and methotrexate with or without IVIG has been effective in prophylaxis against an immune response and in reversing the immune response in CRIM-negative infantile Pompe patients treated with ERT. An ongoing study in treatment-naïve CRIM-negative patients using a similar prophylactic protocol is accruing patients (http:// clinicaltrials.gov, NCT00701129). Rituximab and methotrexate with IVIG should be used in CRIM-negative patients with antibodies to rhGAA and should also be considered for the rare CRIM-positive Pompe patients at risk for a high-titer sustained immune response.

This successful tolerance induction to rhGAA in CRIMnegative Pompe patients will allow for better understanding of the neurological and muscular challenges facing CRIM-negative patientswho survivelonger tolerating theERT. It allows usto compare tolerant CRIM-negative versus CRIM-positive patients for differences, if any, in the central nervous system and other organs. 


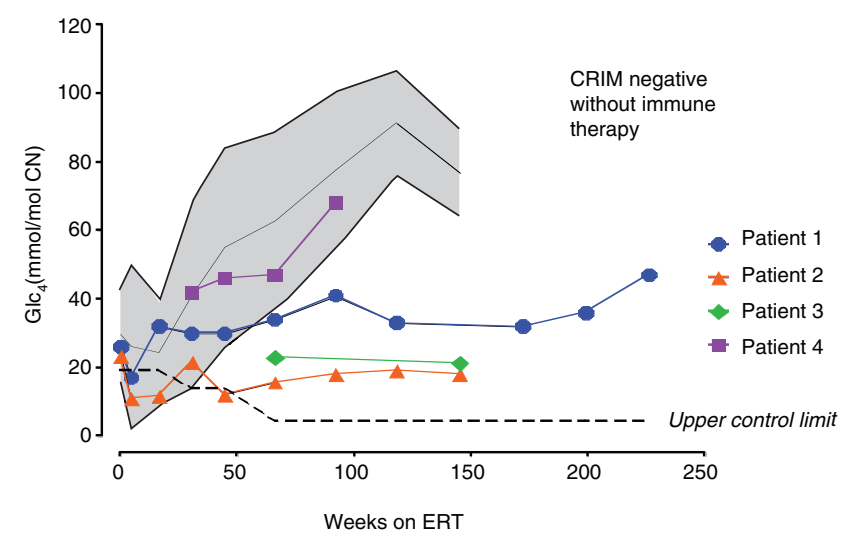

Figure 3 Change in urinary glucose tetrasaccharide $\left(\mathrm{Glc}_{4}\right)$ biomarker concentration on enzyme replacement therapy for the four patients treated by immune therapy. Comparative data for CRIM-negative patients without immune therapy $(n=8, \pm S D)$ are displayed in gray. Mean value of $\mathrm{Glc}_{4}$ over different time periods (bins) was calculated for each patient, and median value for each bin was used to plot the time point. Upper limit of normal controls is represented by dashed line, which is $\leq 4.4 \mathrm{mmol} / \mathrm{mol} \mathrm{CN}$ for more than 1 year of age. Therapeutically treated patient $1 \mathrm{Glc}_{4}$ levels (mean \pm SD: $31 \pm 13 \mathrm{mmol} / \mathrm{mol} \mathrm{CN}, 26$ measurements) and patient $2 \mathrm{Glc}_{4}$ levels (16 \pm $6 \mathrm{mmol} / \mathrm{mol} \mathrm{CN}, 40$ measurements) remained lower than those of nontreated CRIM-negative patients. Similarly, prophylactically treated patient $3 \mathrm{Glc}_{4}$ levels remained lower than those of nontreated CRIM-negative patients $(21,23$ $\mathrm{mmol} / \mathrm{mol} \mathrm{CN}$ ). In contrast, patient 4 had levels that were comparable to those of the nontreated CRIM-negative group ( $50 \pm 14 \mathrm{mmol} / \mathrm{mol} \mathrm{CN}, 8$ measurements). Note that for patients 3 and 4 , last urine samples for $\mathrm{Glc}_{4}$ were available for later time points than anti-acid $\alpha$-glucosidase (GAA) antibody measurement depicted in Figure 2.

This tolerance-inducing strategy might also be more broadly applicable to other conditions treated with therapeutic proteins such as other lysosomal storage diseases, severe hemophilia $\mathrm{A}$ and $\mathrm{B}$, and other conditions in which a foreign protein elicits robust antibody responses that interfere with product efficacy. These lessons may also be important for gene therapy approaches, which may be functionally limited by immune responses.

\section{ACKNOWLEDGMENTS}

We thank the many physicians and staff in each institution who provide ongoing multidisciplinary care to these complex patients. In particular, we are grateful to Stephen C. Kurachek for his wisdom in the management of such complex patients, Sara Froyen-Gernbacher for helping with the initiation of the immunotherapy, Kristi J. Jarvis for help with manuscript preparation, and Erica L. Swift for help with the figures.

\section{DISCLOSURE}

Y.H.M.: Consultant for Genzyme and received honorarium from Genzyme. N.J.M.: Consultant for Genzyme. S.A.J.: Consultant for Genzyme and received honorarium from Genzyme. D.B.: Received honorarium and research funding from Genzyme. L.E.C.: Received honorarium from Genzyme; Research supported by Genzyme, PTC Therapeutics, Leal Foundation, and families of SMA; Received grant support from National Skeletal Muscle Research Center; Member of
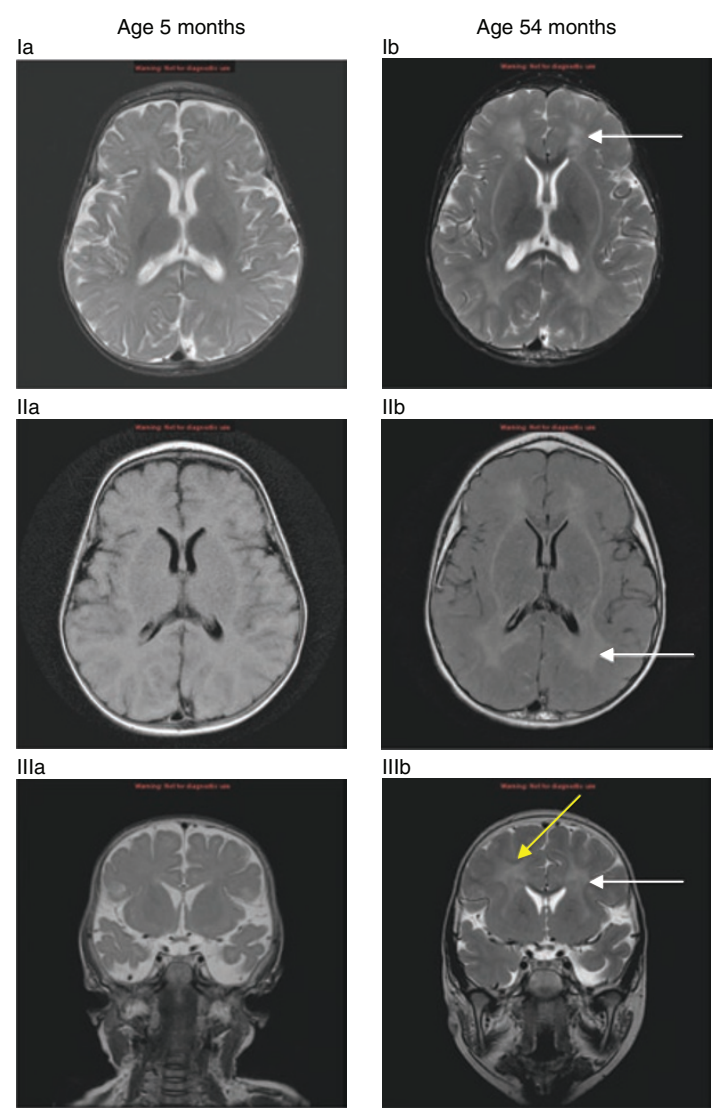

Figure 4 Long-term changes on brain magnetic resonance imaging (MRI) of patient 1. The images on the left, taken at age 5 months, show normal myelination for age. The images on the right, at age 54 months (4.5 years) show development of $\mathrm{T} 2$ prolongation within the deep white matter (white arrows) in the periventricular white matter, centrum semiovale, and external capsule, sparing the subcortical U-fibers (yellow arrow) as well as the internal capsule. I: T2 axial scans at ages (la) 5 months and (Ib) 54 months; II: T2 fast low-angle inversion recovery (FLAIR) images; III: T2 coronal scans.

Pompe Registry Board of Advisors for Genzyme. S.P.Y.: Received honorarium and research funding from Genzyme. P.S.K.: Received honorarium and research funding from Genzyme; Member of Pompe and Gaucher Disease Registry Advisory Boards for Genzyme. W.R.: Received honorarium and clinical trial funding from Genzyme. A.W.: Received honorarium from Genzyme.

\section{REFERENCES}

1. Kishnani PS, Howell RR. Pompe disease in infants and children. J Pediatr 2004;144(suppl 5):S35-S43.

2. Amalfitano A, Bengur AR, Morse RP, et al. Recombinant human acid alphaglucosidase enzyme therapy for infantile glycogen storage disease type II: results of a phase 1/II clinical trial. Genet Med 2001;3:132-138.

3. Kishnani PS, Corzo D, Nicolino M, et al. Recombinant human acid [alpha]glucosidase: major clinical benefits in infantile-onset Pompe disease. Neurology 2007;68:99-109.

4. Kishnani PS, Goldenberg PC, DeArmey SL, et al. Cross-reactive immunologic material status affects treatment outcomes in Pompe disease infants. Mol Genet Metab 2010;99:26-33.

5. Mendelsohn NJ, Messinger YH, Rosenberg AS, Kishnani PS. Elimination of antibodies to recombinant enzyme in Pompe's disease. N Engl J Med 2009;360:194-195. 


\section{ORIGINAL RESEARCH ARTICLE}

6. Kishnani PS, Nicolino M, Voit $T$, et al. Chinese hamster ovary cell-derived recombinant human acid alpha-glucosidase in infantile-onset Pompe disease. J Pediatr 2006;149:89-97.

7. Young SP, Zhang $H$, Corzo D, et al. Long-term monitoring of patients with infantile-onset Pompe disease on enzyme replacement therapy using a urinary glucose tetrasaccharide biomarker. Genet Med 2009;11:536-541.

8. Brooks DA. Immune response to enzyme replacement therapy in lysosomal storage disorder patients and animal models. Mol Genet Metab 1999;68:268-275.

9. Wang J, Lozier J, Johnson G, et al. Neutralizing antibodies to therapeutic enzymes: considerations for testing, prevention and treatment. Nat Biotechnol 2008;26:901-908.

10. Antonarakis SE, Rossiter JP, Young M, et al. Factor VIII gene inversions in severe hemophilia A: results of an international consortium study. Blood 1995;86:2206-2212.

11. Kakkis ED, Muenzer J, Tiller GE, et al. Enzyme-replacement therapy in mucopolysaccharidosis I. N Engl J Med 2001;344:182-188.

12. Linthorst GE, Hollak CE, Donker-Koopman WE, Strijland A, Aerts JM. Enzyme therapy for Fabry disease: neutralizing antibodies toward agalsidase alpha and beta. Kidney Int 2004;66:1589-1595.

13. Warrier I, Ewenstein BM, Koerper MA, et al. Factor IX inhibitors and anaphylaxis in hemophilia B. J Pediatr Hematol Oncol 1997;19:23-27.

14. Wraith JE, Beck M, Lane R, et al. Enzyme replacement therapy in patients who have mucopolysaccharidosis I and are younger than 5 years: results of a multinational study of recombinant human alpha-L-iduronidase (laronidase). Pediatrics 2007;120:e37-e46.

15. Banugaria SG, Prater SN, Ng YK, et al. The impact of antibodies on clinical outcomes in diseases treated with therapeutic protein: lessons learned from infantile Pompe disease. Genet Med 2011;13:729-736.

16. Smith $K G$, Jones RB, Burns SM, Jayne DR. Long-term comparison of rituximab treatment for refractory systemic lupus erythematosus and vasculitis: Remission, relapse, and re-treatment. Arthritis Rheum 2006;54:2970-2982.
17. Chuansumrit A, Husapadol S, Wongwerawattanakoon P, Hongeng S, Sirachainan $\mathrm{N}$, Pakakasama S. Rituximab as an adjuvant therapy to immune tolerance in a haemophilia A boy with high inhibitor titre. Haemophilia 2007;13:108-110.

18. Biss $T$, Velangi MR, Hanley JP. Failure of rituximab to induce immune tolerance in a boy with severe haemophilia A and an alloimmune factor VIII antibody: a case report and review of the literature. Haemophilia 2006;12:280-284.

19. Carcao M, St Louis J, Poon MC, et al. Rituximab for congenital haemophiliacs with inhibitors: a Canadian experience. Haemophilia 2006;12:7-18.

20. Hay C, Recht M, Carcao M, Reipert B. Current and future approaches to inhibitor management and aversion. Semin Thromb Hemost 2006;32 (suppl 2):15-21.

21. Stasi R, Cooper N, Del Poeta G, et al. Analysis of regulatory T-cell changes in patients with idiopathic thrombocytopenic purpura receiving B cell-depleting therapy with rituximab. Blood 2008;112:1147-1150.

22. Rohrbach M, Klein A, Köhli-Wiesner A, et al. CRIM-negative infantile Pompe disease: 42-month treatment outcome. J Inherit Metab Dis 2010;33:751-757.

23. Nimmerjahn F, Ravetch JV. Anti-inflammatory actions of intravenous immunoglobulin. Annu Rev Immuno/ 2008;26:513-533.

24. Anthony RM, Wermeling F, Karlsson MC, Ravetch JV. Identification of a receptor required for the anti-inflammatory activity of IVIG. Proc Natl Acad Sci USA 2008;105:19571-19578.

25. Carson KR, Focosi D, Major EO, et al. Monoclonal antibody-associated progressive multifocal leucoencephalopathy in patients treated with rituximab, natalizumab, and efalizumab: a Review from the Research on Adverse Drug Events and Reports (RADAR) Project. Lancet Oncol 2009;10:816-824.

26. Joseph A, Munroe K, Housman M, Garman R, Richards S. Immune tolerance induction to enzyme-replacement therapy by co-administration of short-term, low-dose methotrexate in a murine Pompe disease model. Clin Exp Immunol 2008;152:138-146.

27. Desnick RJ. Enzyme replacement and enhancement therapies for lysosomal diseases. J Inherit Metab Dis 2004;27:385-410. 